\title{
The dark secrets of gaseous nebulae - highlights from deep spectroscopy
}

\author{
Xiaowei Liu \\ Professor of Astronomy \\ Kavli Institute for Astronomy and Astrophysics, Peking University
}

September 9, 2021 


\section{Chapter 1}

\section{The dark secrets of gaseous nebulae}

\subsection{Emission line nebulae}

The existence and distribution of the chemical elements and their isotopes is a consequence of nuclear processes that have taken place in the past in the Big Bang and subsequently in stars and in the interstellar medium (ISM) where they are still ongoing (Pagel, 1997). A large body of our knowledge of the distribution and production of elements in the universe rests on observations and analyses of photoionized gaseous nebulae. Ionized and heated by strong ultraviolet (UV) radiation fields, photoionized gaseous nebulae glow by emitting strong emission lines (Osterbrock and Ferland, 2005). They are therefore also commonly named emission line nebulae.

Examples of emission line nebulae include $\mathrm{H}$ II regions, planetary nebulae (PNe) and the broad and narrow emission line regions found in active galactic nuclei (Fig.11.1). H II regions are diffuse nebulae found around newly formed young, massive stars and trace the current status of the ISM. Giant extragalactic H II regions, sign posts of massive star formation activities, are amongst the most prominent features seen in a gas-rich, star-forming galaxy. In some galaxies, the star forming activities are so intense that the whole galaxy becomes a giant H II region. Such galaxies are called H II or starburst galaxies and are observable to 

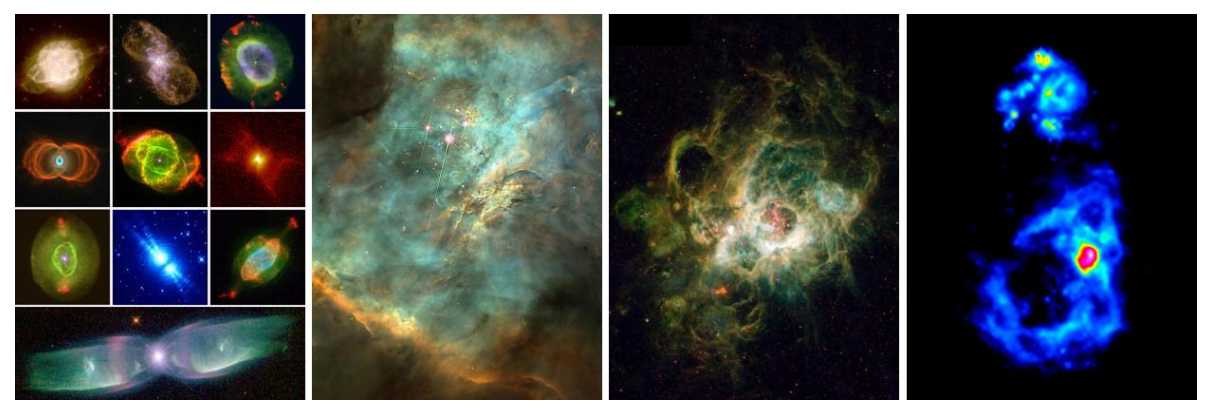

Figure 1.1: Examples of emission line nebulae. From left to right: a) PNe (HST images obtained by B. Balick and collaborators; c.f. http://www.astro.washington.edu/users/balick/WFPC2/index.html); b) The H II region M 42 (the Orion Nebula; Hubble Heritage image obtained by C. R. O'Dell and S. K. Wong, c.f. http://hubblesite.org/newscenter/archive/releases/1995/45); c) NGC 604, a giant extragalactic H II region in the outskirts of the Local Group spiral galaxy M 33 (Hubble Heritage image obtained by H. Yang, c.f. http://heritage.stsci.edu/2003/30/supplemental.html; d) The starburst galaxy IZw 18 (based on HS'T/WFPC2 data obtained by E. Skillman). The linear sizes of these objects differ vastly, ranging from about a tenth of a parsec $(1 \mathrm{pc}=$ $\left.3.262 \mathrm{lyr}=3.086 \times 10^{16} \mathrm{~m}\right)$ for PNe and M 42 to several hundred parsecs for NGC 604 and $\mathrm{IZw} 18$.

large cosmic distances.

PNe are amongst the most beautiful objects in the sky and, arguably, the queen of the night. They were given the name by William Herschel (Herschel, 1785) based on their distinct structures and, for some of them, nearly circular and overall uniform appearances resembling the greenish disk of a planet. They have however nothing to do with a planet (but see later) and are in fact expanding gaseous envelopes expelled by low- and intermediate-mass stars in late evolutionary stage after the exhaustion of central nuclear fuel at the end of the asymptotic giant branch (AGB) phase. Owing to their relatively simple geometric structure, a nearly symmetric shell of gas ionized by a single, centrally located white dwarf, PNe are ideal cosmic laboratories to study the atomic and radiative processes governing cosmic low density plasmas. PNe have played and continue to play a central role in formulating the theory of photoionized gaseous nebulae. Representing a pivotal, albeit transient evolutionary phase of low- and intermediate-mass stars (the overwhelming majority in a galaxy), PNe play a major role in the galactic ecosystem - in the constant enrichment of metals, formation and destruction of molecules and dust grains and in the recycling of gas in the ISM. Today, studies of PNe have gone far beyond the objects themselves. PNe are widely used to trace the kinematics of the host galaxies and the intracluster stellar populations. They have even 


\subsection{THE FOUNDING OF THE THEORY OF PHOTOIONIZED GASEOUS NEBULAE3}

been successfully utilized to measure the Hubble constant of the cosmic expansion.

Recent progress in observational techniques, atomic data and high-performance computation have enabled reliable measurements and analyses of lines as faint as one millionth of $\mathrm{H} \beta$, including weak optical recombination lines (ORLs) from abundant heavy elements $(\mathrm{C}, \mathrm{N}, \mathrm{O}$, $\mathrm{Ne}$ and $\mathrm{Mg}$ ) and collisionally excited lines (CELs) from rare elements, such as fluorine and s- and r-process elements. This allows one to address some of the long-standing problems in nebular astrophysics as well as opening up new windows of opportunity.

In this contribution, I will briefly review the development of the theory of photoionized gaseous nebulae, highlighting some of the key events. I will then present some recent developments of deep spectroscopy of PNe and $\mathrm{H}$ II regions, concentrating on observations of faint heavy element ORLs. I will show that there is strong evidence that nebulae contain another previously unknown component of cold (about 1,000 K), high-metallicity plasma, probably in the form of H-deficient inclusions embedded in the warm (about 10,000 K) diffuse nebula of "normal (i.e. near solar ) composition". This cold gas emits essentially all the observed flux of heavy element ORLs, but is too cool to excite any significant optical or ultraviolet CELs and thus invisible via the latter. The existence of H-deficient gas in PNe and probably also in $\mathrm{H}$ II regions, not predicted by the current stellar evolution theory, provides a natural solution to the long-standing dichotomy between nebular plasma diagnostics and abundance determinations using CELs on the one hand and ORLs on the other, a discrepancy that is ubiquitously observed in Galactic and extragalactic PNe as well as H II regions.

\subsection{The founding of the theory of photoionized gaseous nebulae}

The applications of the principle of "Chemical analysis by observations of spectra" expounded by G. Kirchhoff and R. W. Bunsen (1860) by W. Huggins and W. A. Millar to the analyses of stellar and nebular spectra in 1860s heralded the rise of astrophysics. In an accompanying paper to their monumental work on stellar spectra, Huggins and Millar presented their first visual spectroscopic observations of eight PNe, including the Cat's Eye Nebula NGC 6543 in Draco (Huggins and Millar, 1864). Instead of the dark (absorption) 
Fraunhofer lines observed in the spectra of the Sun and other stars, they found bright emission lines in the nebular spectra and concluded that the nebulae must consist of "enormous masses of luminous gas or vapour". While the Fraunhofer $\mathrm{F}$ line (H $\beta$ at $4861 \AA$ ) did appear in emission, the two bright, nearby lines $\lambda \lambda 4959,5007$ were not Fraunhofer lines at all. The availability of dry photographic plates (light-sensitive silver bromide salts held in a gelatine emulsion on the glass), replacing the old wet collodion plates, made it possible to record long exposures of nebular spectra and more nebular emission lines were revealed in the blue and ultraviolet (UV) wavelength regions. In 1882, Huggins successfully photographed a UV spectrum of the nearest bright H II region, the Orion Nebula, and detected a strong emission line near $3728 \AA$ (Huggins, 1882).

By the late 1920s, dozens of nebular lines had been detected and their wavelengths accurately measured (Wright, 1918), yet most of them remained unidentified and were attributed to some hypothetical element "nebulium". The big breakthrough in understanding nebular spectra came in 1927 from Ira Bowen who, stimulated by a heuristic speculation by H. N. Russell that the nebulium lines must be due to abundant "atoms of known kinds shining under unfamiliar conditions" such as in gas of very low density (Russell, Dugan and Stewart, 1927), identified eight of the strongest nebular lines as being due to the forbidden transitions from the low excitation meta-stable states of the ground electron configurations of singly ionized oxygen, nitrogen and doubly ionized oxygen (Bowen, 1927a, b, c). For the first time, the physical processes in gaseous nebulae could be understood. This important discovery paved the way for future studies of nebular structure and chemical composition.

Great strides forward in nebular spectral observations were made starting in 1910 and through the 1930s, powered by a number of technological inventions, such as the Schmidt camera (Schmidt, 1932), the high-efficiency blazed diffraction grating (Wood, 1910) and the image slicer (Bowen, 1938). Deep exposures revealed faint lines from the refractory elements such as potassium, calcium, silicon, magnesium and iron, demonstrating that nebulae are qualitatively made of similar material as stars (Bowen and Wyse, 1939). In the following three decades, the structures and the underlying physics governing photoionized gaseous nebulae had been worked out quantitatively, including photoionization and recombination (Zanstra, 1926, 1927; Strömgren, 1939), heating and cooling (Zanstra, 1931; Baker, Menzel 
and Aller, 1938; Aller, Baker and Menzel, 1939; Spitzer, 1948), recombination and collisional excitation (Baker and Menzel, 1938; Menzel, Aller and Hebb, 1941; Seaton, 1954; Burgess, 1958; Seaton, 1959; Seaton and Osterbrock, 1957), and elemental abundance determinations (Bowen and Wyse, 1939; Menzel and Aller, 1941; Wyse 1942; Aller and Menzel, 1945). Origin of PNe, as descendants of red giant stars was also understood (Shklovsky, 1956). Photoionization models incorporating all known physics were constructed (Hjellming, 1966; Goodson, 1967; Harrington, 1968; Rubin 1968; Flower, 1969) and the models reproduced observations well. The theory of photoionized gaseous nebulae as it stood in late 1960s was nicely summarized in Astrophysics of Gaseous Nebulae by Donald Edward Osterbrock (1974).

\subsection{CELs and ORLs - the dichotomy}

While the theory seemed well established and solid, there were dark clouds hovering on the horizon. One concerned the measurement and interpretation of weak nebular emission lines, and the other regarded the possible presence of significant temperature inhomogeneities in nebulae and their effects on nebular abundance determinations.

Except for a few lines excited under specific environments, such as the Bowen fluorescence lines (e.g. O III $\lambda \lambda 3133,3341,3444$; Bowen, 1934, 1935), strong lines radiated by photoionized gaseous nebulae fall into two categories, recombination lines (RLs) and CELs. Hydrogen and helium ions, the main baryonic components of an ionized gaseous nebula, capture free electrons and recombine, followed by cascades to the ground state. During this process, a series of RLs are radiated (e.g. $\mathrm{H} \alpha \lambda 6563, \mathrm{H} \beta \lambda 4861$, He I $\lambda \lambda 4472,5876,6678$ and He II $\lambda 4686$ ). The ground electron configuration of multi-electron ions of heavy elements yields some low excitation energy levels (within a few $\mathrm{eV}$ from the ground state, such as $\mathrm{O}^{++} 2 \mathrm{p}^{2}{ }^{3} \mathrm{P}_{0,1,2}^{\mathrm{o}},{ }^{1} \mathrm{D}_{2}^{\circ},{ }^{1} \mathrm{~S}_{0}^{\circ}$, which can be excited by impacts of thermal electrons, which have typical energies of $\sim 1 \mathrm{eV}$ in photoionized gaseous nebulae of solar composition. Follow-up de-excitation by spontaneous emission yields the so-called CELs. Quite often, those lines are electron dipole forbidden transitions, so they are commonly called forbidden lines (e.g. 
[O III] collisionally excited lines

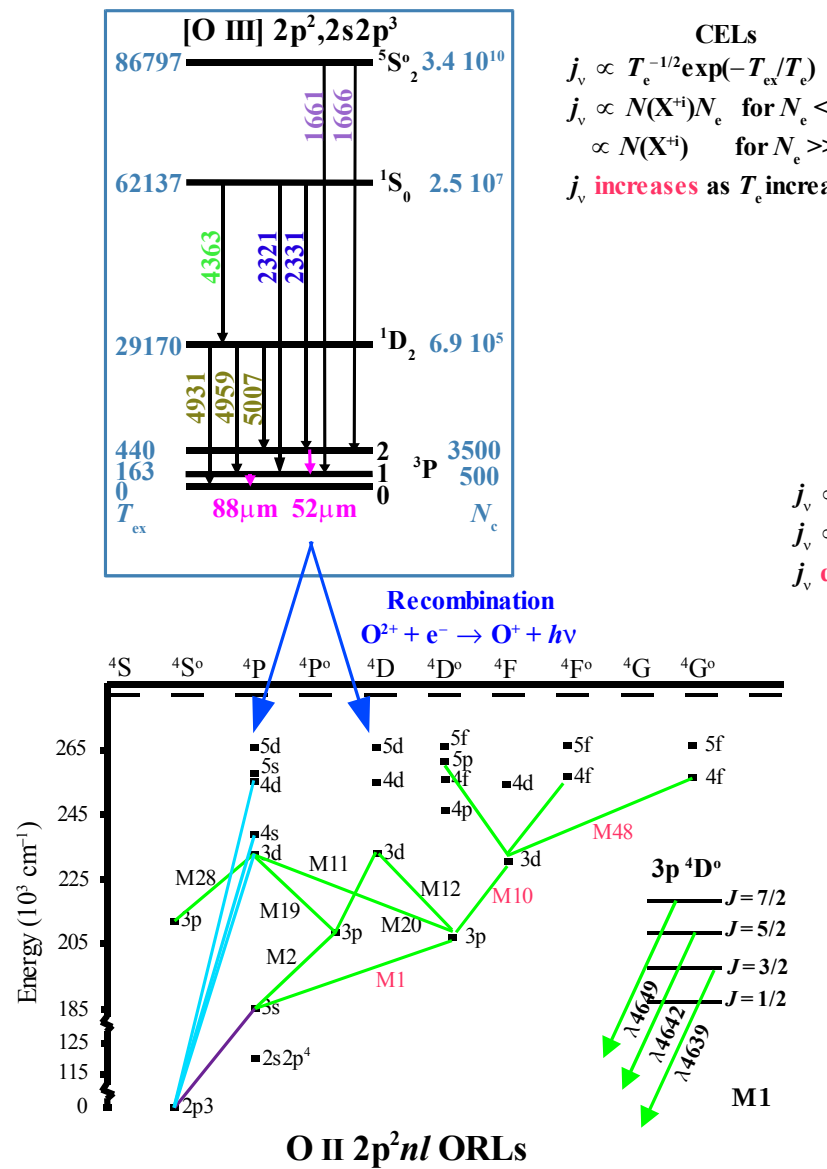

\section{H I recombination lines/continua}
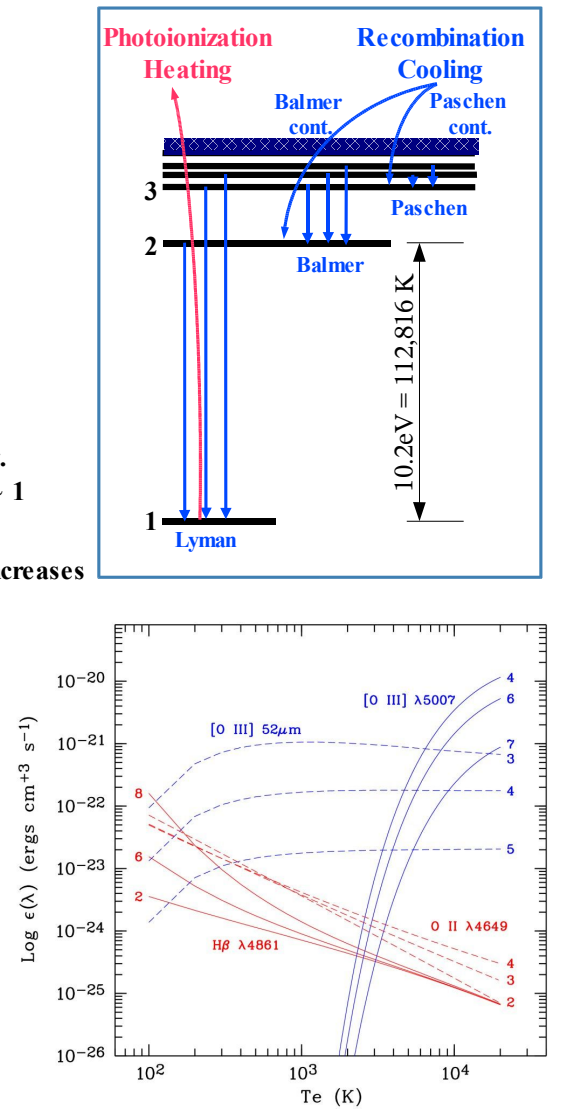

Emissivities per ion per electron

Figure 1.2: Top-left: Schematic diagram showing the four lowest spectral terms of $\mathrm{O}^{++}$ formed by the $2 \mathrm{p}^{2}$ ground and $2 \mathrm{p}^{3}$ electron configurations. The levels are labeled with excitation energy $T_{\mathrm{ex}}$ (in Kelvin) and critical density $N_{\mathrm{c}}\left(\mathrm{in}_{\mathrm{cm}}^{-3}\right)$; Bottom-left: Schematic Grotrian diagram of $\mathrm{O}$ II, illustrating the $\mathrm{O}$ II recombination line spectrum produced by recombination of $\mathrm{O}^{++}$; Top-right: Photoionization and recombination of hydrogen. Photoionization heats the gas whereas recombination cools the gas; Bottom-right: Emissivities of the collisionally excited [O III] $\lambda 5007$ optical forbidden line and $52 \mu \mathrm{m}$ far-IR fine-structure line, and of the recombination lines $\mathrm{H} \beta \lambda 4861$ and $\mathrm{O}$ II $\lambda 4649$, as a function of electron temperature $T_{\mathrm{e}}$ for selected electron densities. The curves are labeled with logarithms of electron densities. Emissivities of recombination lines depend only weakly on electron density under typical (low-density) nebular conditions. 
[O II] $\lambda \lambda 3726,3729,[\mathrm{O}$ III] $\lambda \lambda 4959,5007,[\mathrm{~N} \mathrm{II}] \lambda \lambda 6548,6584$ and [Ne III] $\lambda \lambda 3868,3967)$. Recombination of free electrons to bound states of hydrogen and helium ions also yields weak nebular continuum emission. For example, recombination to the H I $n=2$ state yields the near UV nebular continuum Balmer discontinuity at $\lambda<3646 \AA$. Recombination of heavy element ions, followed by cascading to the ground state, also produces RLs. However, for typical cosmic composition, i.e. approximately solar, even the most abundant heavy element oxygen has a number density less than one thousandth of hydrogen. Thus heavy element RLs are much weaker, at the level of a few thousandth of $\mathrm{H} \beta$ or less, and are observable generally only in the optical. Those lines are therefore often called ORLs. Sample ORLs from abundant heavy element ions that have been well studied and discussed in the current contribution include C II $\lambda 4267$, C III $\lambda 4649, \mathrm{~N}$ II $\lambda 4041$, O I $\lambda 7773$, O II $\lambda 4089$, O III $\lambda 3265$, Ne II $\lambda 4392$ and Mg II $\lambda 4481$.

Except for IR fine-structure lines arising from ground spectral terms, emissivities of CELs have an exponential dependence on electron temperature, $T_{\mathrm{e}}, \epsilon\left(\mathrm{X}^{+\mathrm{i}}, \lambda\right) \propto T_{\mathrm{e}}^{-1 / 2} \exp \left(-E_{\mathrm{ex}} / k T_{\mathrm{e}}\right)$ (the Boltzmann factor), where $E_{\text {ex }}$ is excitation energy of the upper level of transition emitting wavelength $\lambda$ by ion $\mathrm{X}^{+\mathrm{i}}$ following collisional excitation by electron impacts (Fig. 1.2). At low densities, $N_{\mathrm{e}}<<N_{\mathrm{c}}$, i.e. electron density $N_{\mathrm{e}}$ much lower than the upper level's critical density $N_{\mathrm{c}}$ (for any energy level above the ground, a critical density $N_{\mathrm{c}}$ can be defined such that above which collisional de-excitation dominates over spontaneous radiative decay in de-populating the level; c.f. Osterbrock \& Ferland, 2005), we have $\epsilon\left(\mathrm{X}^{+\mathrm{i}}, \lambda\right) \propto N\left(\mathrm{X}^{+\mathrm{i}}\right) N_{\mathrm{e}}$, where $N\left(\mathrm{X}^{+\mathrm{i}}\right)$ is number density of ion $\mathrm{X}^{+\mathrm{i}}$. At high densities, $N_{\mathrm{e}}>>N_{\mathrm{c}}$, emission of CELs are suppressed by collisional de-excitation and $\epsilon\left(\mathrm{X}^{+\mathrm{i}}, \lambda\right) \propto N\left(\mathrm{X}^{+\mathrm{i}}\right)$. Unlike CELs, emissivities of RLs increase with decreasing $T_{\mathrm{e}}$ by a power law. Under typical nebular conditions $\left(N_{\mathrm{e}}<<10^{8} \mathrm{~cm}^{-3}\right), \epsilon\left(\mathrm{X}^{+\mathrm{i}}, \lambda\right) \propto T_{\mathrm{e}}^{-\alpha} N\left(\mathrm{X}^{+\mathrm{i}+1}\right) N_{\mathrm{e}}$, where $\alpha \sim 1$ and $N\left(\mathrm{X}^{+\mathrm{i}+1}\right)$ is number density of the recombining ion $\mathrm{X}^{+\mathrm{i}+1}$.

In a ground breaking work, Arthur B. Wyse published very deep spectra that he obtained with I. S. Bowen using the Lick 36-inch reflector for a number of bright PNe (Wyse, 1942). About 270 spectral lines were detected. Many were weak permitted transitions from abundant C, N and O ions. In the Saturn Nebula NGC 7009 alone, "... more than twenty lines or blends of O II have been observed, free of blending with lines of other elements. There 
are good grounds for the assumption, previously made, that these lines originate in electron captures by $\mathrm{O}$ III ions, just as the Balmer lines originate in electron captures by $\mathrm{H}$ II ions, and that therefore the relative intensities of the oxygen and hydrogen lines give a measure of the rates of recombination, and therefore of the relative abundance, of the two kinds of ions .... The important thing is that for this nebula a sufficient number of oxygen lines has been observed to eliminate all doubt of the correctness of their identifications, even though they are very faint; and also that they permit the direct estimate of the relative abundance of $\mathrm{H}$ II and $\mathrm{O}$ III ions, ..., by a method that is relatively independent of assumptions regarding electron density and velocity distribution or their variations throughout the nebula."

From the $\mathrm{O}$ II ORL strengths, Wyse deduced $\mathrm{O} / \mathrm{H}$ abundances which were much higher than the values obtained by Menzel and Aller (1941) from analyses of the [O III] forbidden lines. He concluded "The discrepancy, then, between the relative abundance found by Menzel and Aller, on the one hand, and from the recombination spectra, on the other, is of the order of 50 for NGC 7027 and of 500 for NGC 7009." Shortly after the publication of this prophetic article, Wyse was called to serve in the War and died tragically on duty the night of June 8, 1942 in a disastrous accident over the Atlantic Ocean, off the New Jersey coast, at the age of 17 days shy of 33 .

As stressed by Wyse, ionic abundances deduced from intensities of heavy element ORLs relative to $\mathrm{H} \beta$, a method based on comparing lines of like to like, have the advantage that they are almost independent of the nebular thermal and density structures. In contrast, ionic abundances deduced from the intensity ratio of the collisionally excited, much stronger [O III] $\lambda \lambda 4959,5007$ forbidden lines relative to $\mathrm{H} \beta$, have an exponential dependence on the adopted nebular electron temperature. Theoretically, ionic abundances deduced from heavy element ORLs should thus be more reliable, provided the lines can be measured accurately. Unfortunately, latter development showed that accurate flux measurements for faint nebular emission lines were NOT possible after all with the technique available then, i.e. spectrophotography, due to the non-linearity of photographic plates. Via detailed comparisons between the observed fluxes of $\mathrm{H}$ I and He II RLs and continua and those predicted by the recombination theory, it became clear that spectrophotographic observations systematically overestimated intensities of faint lines, by as much as over a factor of ten (Seaton, 
1960; Kaler, 1966; Miller, 1971; Miller and Mathews, 1972). That spectrophotographic measurements of faint lines cannot be trusted seemed to be further supported by work in the 1980s that contrasted $\mathrm{C}^{++} / \mathrm{H}^{+}$ionic abundances deduced from the collisionally excited C III] $\lambda \lambda 1907,1909$ intercombination lines and from the faint C II $\lambda 4267$ ORL (c.f. Barker, 1991 and references therein), suggesting that the intensity of the faint C II $\lambda 4267$ line had either not been interpreted correctly or been grossly overestimated (Rola and Stasińska, 1994).

In another worrisome development, Manual Peimbert showed that if nebulae are nonisothermal and have (localized and random) temperature fluctuations, then $T_{\mathrm{e}}$ deduced from the $[\mathrm{O}$ III $]$ nebular to auroral line intensity ratio $(\lambda 4959+\lambda 5007) / \lambda 4363$ will overestimate the average emission temperature of the $\lambda \lambda 4959,5007$ nebular lines and of $\mathrm{H} \beta$ at $4861 \AA$, leading to underestimated $\mathrm{O}^{++} / \mathrm{H}^{+}$ionic abundance ratio deduced from the $(\lambda 4959+\lambda 5007) / \mathrm{H} \beta$ ratio (Peimbert, 1967). He presented evidence pointing to the presence of large temperature fluctuations in $\mathrm{PNe}$ and $\mathrm{H}$ II regions by finding that $T_{\mathrm{e}}$ 's derived from the Balmer jump, $T_{\mathrm{e}}(\mathrm{BJ})$, are systematically lower than those derived from the [O III] forbidden line ratio, $T_{\mathrm{e}}([\mathrm{O}$ III $])$ (Peimbert 1967; Peimbert, 1971). Given the weakness of nebular continuum emission, measuring the Balmer jump accurately was no easy task and his results were disputed (Barker, 1978). Theoretically, while some systematic spatial temperature variations undoubtedly occur within a nebula, due to changes of ionization structure and cooling rates as a function of position induced by varying ionization radiation field and density distribution, no mechanisms are known capable of generating large, localized temperature fluctuations, certainly nothing of the magnitude implied by Peimbert's measurements, which yield a typical value of 0.055 for the temperature fluctuation parameter, $t^{2}$, or fluctuations of an amplitude of 23 per cent.

The advent of linear, high quantum efficiency, large dynamic range and large format CCDs in the 1980s made it possible for the first time to obtain reliable measurements of faint emission lines for bright nebulae. Meanwhile, the completion of the Opacity Project (Seaton, 1987; Cunto et al., 1993) has allowed the atomic data necessary to analyze those spectral features, specifically their effective recombination coefficients, to be calculated with high accuracy. Liu and Danziger (1993) obtained CCD measurements of the Balmer jump for a sample of $\mathrm{PNe}$ and found that $T_{\mathrm{e}}(\mathrm{BJ})$ is indeed systematically lower than $T_{\mathrm{e}}([\mathrm{O}$ III $])$ 
obtained for the same object. They deduced that on average, $t^{2}=0.035$, smaller than that found earlier by Peimbert (1971) but still significant, enough to cause the $\mathrm{O}^{++} / \mathrm{H}^{+}$ abundance ratio derived from the $\lambda \lambda 4959,5007$ forbidden lines to be underestimated by a factor of two.

Liu et al. (1995) presented high quality IPCS and CCD optical spectrophotometry for the legendary Saturn Nebula NGC 7009 as well as new effective recombination coefficients for O II ORLs. Nearly a hundred O II ORLs were measured, yielding an $\mathrm{O}^{++} / \mathrm{H}^{+}$ionic abundance that is consistently higher, by a factor of $\sim 4.7$, than the value deduced from the strong [O III] forbidden lines $\lambda \lambda 4959,5007$. The close agreement of results deduced from a large number of O II ORLs from a variety of multiplets of different multiplicities, parities and electron configurations, vindicates the reliability of the recombination theory and rules out measurement uncertainties ${ }^{1}$ or other effects, such as reddening corrections, line blending or contamination of ORLs by other excitation mechanisms (e.g. fluorescence or charge transfer reactions) as the cause of the large discrepancy between the ORL and CEL abundances. Further analysis of the carbon, nitrogen recombination spectra and of the neon recombination spectrum (Luo et al., 2001) show that in NGC 7009, abundances of these elements derived from ORLs are all higher than the corresponding CEL values by approximately a factor of 5 . If one defines an abundance discrepancy factor (adf) as the ratio of ionic abundances $\mathrm{X}^{\mathrm{i}+} / \mathrm{H}^{+}$deduced from ORLs and from CELs, then in NGC 7009 adf $\sim 5$ for all the four abundant second-row elements of the periodic table: $\mathrm{C}, \mathrm{N}, \mathrm{O}$ and Ne. In NGC 7009, the Balmer discontinuity of the hydrogen recombination spectrum yields $T_{\mathrm{e}}(\mathrm{BJ})$ $=8,100 \mathrm{~K}$, about $2,000 \mathrm{~K}$ lower than forbidden line temperature $T_{\mathrm{e}}([\mathrm{O} \mathrm{III}])=10,100 \mathrm{~K}(\mathrm{Liu}$ et al., 1995). The difference yields a Peimbert's $t^{2}$ value of 0.04 , or temperature fluctuations of an amplitude of 20 per cent.

\footnotetext{
${ }^{1}$ Mathis and Liu (1999) analyzed the observed relative intensities of the [O III] $\lambda \lambda 4959,5007$ and the much fainter $\lambda 4931$ nebular lines and demonstrated that accurate measurements have been achieved over a dynamic range of 10,000.
} 

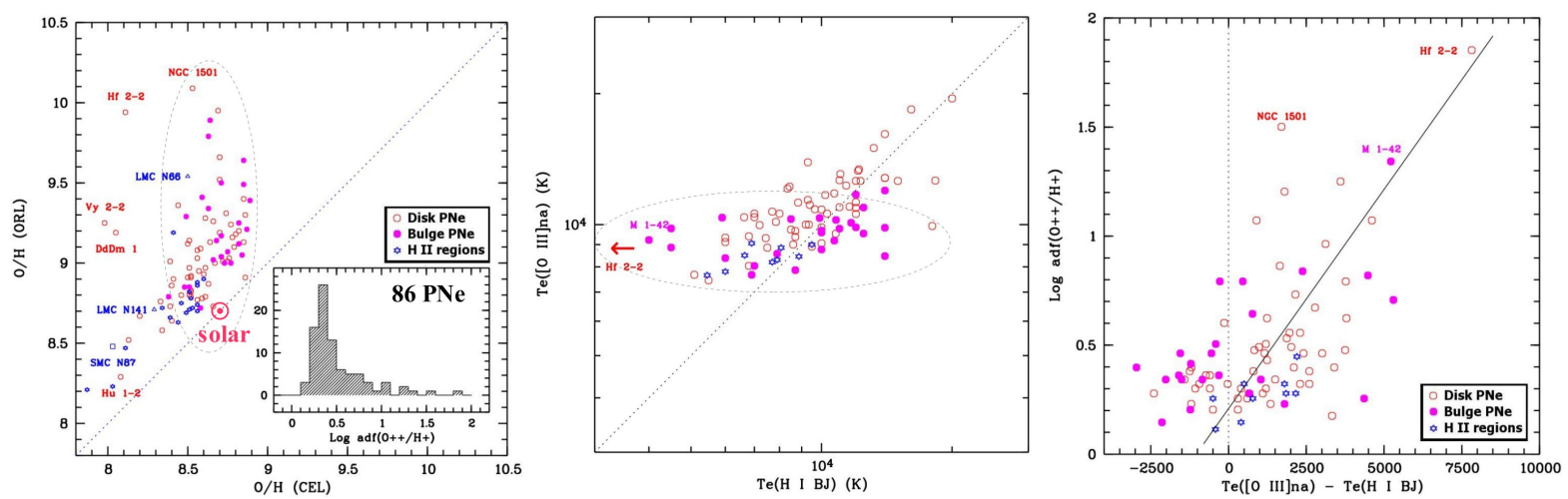

Figure 1.3: Left: $\mathrm{O} / \mathrm{H}$ abundances deduced from ORLs plotted against those derived from CELs. The diagonal dotted line denotes $x=y$. The insert is a histogram of $\operatorname{adf}\left(\mathrm{O}^{++} / \mathrm{H}^{+}\right)$. In the scenario of single composition nebulae (c.f. next section for an alternative interpretation), uncertainties in $\mathrm{O} / \mathrm{H}$ abundances, caused by observational and interpretive errors (e.g. atomic data) are expected to be typically less than 0.05 and 0.1 dex, respectively, for CEL and ORL results; Middle: $T_{\mathrm{e}}\left([\mathrm{O}\right.$ III $]$ ) versus $T_{\mathrm{e}}(\mathrm{BJ})$. Typical uncertainties of $T_{\mathrm{e}}([\mathrm{O}$ III $])$ and $T_{\mathrm{e}}(\mathrm{BJ})$ are 5 and 10 per cent, respectively. The diagonal dotted line denotes $x=y$. With $T_{\mathrm{e}}(\mathrm{BJ})=900 \mathrm{~K}$ and $T_{\mathrm{e}}([\mathrm{O} \mathrm{III}])=8820 \mathrm{~K}$, Hf $2-2$ falls off the left boundary of the plot; Right: $\log \operatorname{adf}\left(\mathrm{O}^{++} / \mathrm{H}^{+}\right)$plotted against $T_{\mathrm{e}}([\mathrm{O} \mathrm{III}])-T_{\mathrm{e}}(\mathrm{BJ})$. The solid line denotes a linear fit obtained by Liu et al. (2001b), prior to the discovery of the very large adf in Hf 2-2. (Adapted from Liu, 2006a)

\subsection{Deep spectroscopic surveys of ORLs}

Is the dichotomy observed in NGC 7009 between ORLs and CELs for nebular plasma diagnostics and abundance determinations ubiquitous amongst emission line nebulae? What is the range and distribution of adf's for individual elements? Are there any correlations between the adf and other nebular properties (morphology, density, temperature, abundance, and age etc.) or properties of the ionizing source? What are the physical causes of the dichotomy? To address those issues, several deep ORL spectroscopic surveys of faint heavy element ORLs have been carried out. So far over a hundred Galactic PNe have been surveyed, plus dozens of Galactic and extragalactic H II regions (e.g. Esteban et al., 2002; Tsamis et al., 2003a,b, 2004; Liu et al., 2004a,b; Wesson et al., 2005; Wang and Liu, 2007; For a complete list of references, please refer to a recent review by Liu, 2006a). Detailed comparisons contrasting ORL and CEL analyses show that (Fig.1.3; c.f. also Liu, 2006a):

1) Ionic abundances deduced from ORLs are always higher than CEL values, i.e. adf $\geq 1$. Adf peaks at 0.35 dex but with a tail extending to much higher values. About 20 and 10 

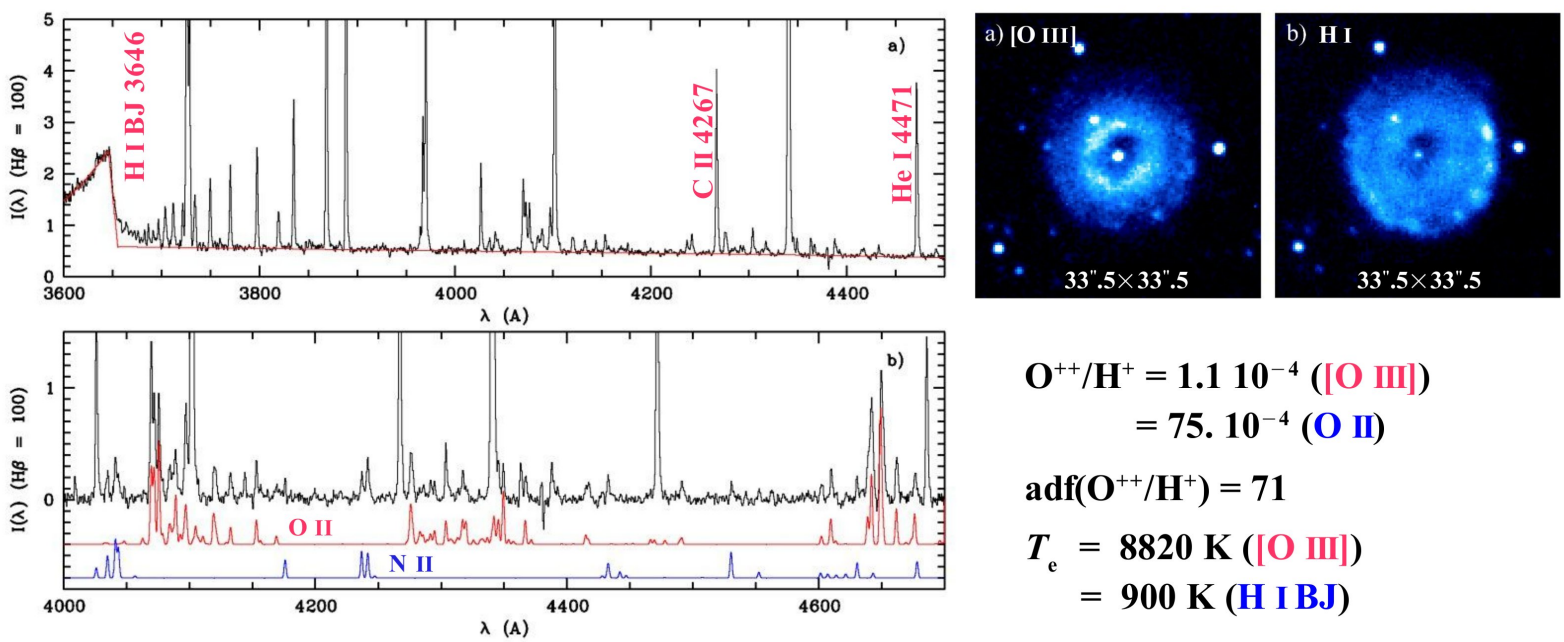

$$
\begin{aligned}
& \mathrm{O}^{++} / \mathrm{H}^{+}=1.1 \mathbf{1 0}^{-4}([\mathrm{O} \mathrm{IIII}]) \\
& =75.10^{-4}(\mathrm{O} \text { II }) \\
& \operatorname{adf}\left(\mathrm{O}^{++} / \mathbf{H}^{+}\right)=71 \\
& T_{\mathrm{e}}=8820 \mathrm{~K}([\mathrm{O}[\mathrm{IIIT}]) \\
& =900 \mathrm{~K}(\mathrm{H} \mathrm{I} \mathrm{BJ})
\end{aligned}
$$

Figure 1.4: Left: The optical spectrum of $\mathrm{Hf} 2-2$, which shows a record $\operatorname{adf}\left(\mathrm{O}^{++} / \mathrm{H}^{+}\right)=71$ and an extremely low $T_{\mathrm{e}}(\mathrm{BJ})$ of $900 \mathrm{~K}$. In the lower panel, also shown are two synthetic recombination line spectra of $\mathrm{O}$ II and N II, respectively. Right: Monochromatic images of Hf 2-2 in the light of [O III] $\lambda 5007$ and $\mathrm{H} \alpha$ 26563 , respectively. (Adapted from Liu et al., 2006)

per cent of nebulae exhibit adf's higher than 5 and 10, respectively. For example, in the bright Galactic disk PN NGC 6153, adf = 9.2 (Liu et al., 2000), whereas in the bulge PN M 1-42, adf $=22$ (Liu et al., 2001b). In the most extreme object discovered so far, Hf 2-2, $\operatorname{adf}\left(\mathrm{O}^{++} / \mathrm{H}^{+}\right)$reaches a record value of 71 (Fig. 1.4);

2) While adf varies from object to object, for a given nebula, C, N, O and Ne all exhibit comparable adf's (thus both CEL and ORL analyses yield compatible abundance ratios, such as $\mathrm{C} / \mathrm{O}, \mathrm{N} / \mathrm{O}$ and $\mathrm{Ne} / \mathrm{O}$, provided lines of the same type, ORLs or CELs, are used for both elements involved in the ratio). Objects showing large adf's also tend to have high helium (ORL) abundances. However, magnesium, the only third-row element which has been analyzed using an ORL, shows no enhancement, even in high-adf objects (Barlow et al., 2003);

3) Excluding metal-poor nebulae in the Galactic halo and in the Large and Small Magellanic Clouds (LMC and SMC, respectively), oxygen abundances deduced from CELs for Galactic H II regions and PNe fall in a narrow range compatible with the solar value. In contrast, ORLs yield much higher abundances, more than ten times solar in some cases. 
4) Similarly, while the [O III] forbidden line ratio yields values of $T_{\mathrm{e}}$ in a narrow range around $10,000 \mathrm{~K}$, as one expects for a photoionized gaseous nebula of solar composition, the Balmer discontinuity yields some very low temperatures, down to below 1,000 K. In fact, the discrepancies in temperature and abundance determinations, using ORLs/continua on the one hand and CELs on the other, seem to be correlated - objects showing large adf's also exhibit very low $T_{\mathrm{e}}$ 's;

5) Large, old PNe of low surface brightness tend to show higher adf's. In addition, spatially resolved analyses of a limited number of bright, extended nebulae of large adf's show that ORL abundances increase towards the nebular center, leading to higher adf's near the center.

\subsection{Evidence of cold, H-deficient inclusions}

What causes the ubiquitous, often alarmingly large, discrepancies between the ORL and CEL plasma diagnostics and abundance determinations? Does the dichotomy imply that there are fundamental flaws in our understanding of the nebular thermal structure, or that we do not even understand basic processes such as the recombination of hydrogenic ions?

Can it be temperature fluctuations as originally postulated by Peimbert (1967)? This conjecture implicitly assumes that the higher abundances yielded by ORLs represent the true nebular composition as they are insensitive to temperature and temperature fluctuations. The discovery of nebulae exhibiting extreme values of adf and the abnormally high metal abundances implied by the observed strengths of ORLs casts however serious doubt on this paradigm. Given that PNe are descendants of low- and intermediate-mass stars, the very high ORL oxygen abundances recorded in objects of extreme adf's, if real and representative of the whole nebula, are extremely difficult to understand in the current theory of stellar evolution and nucleosynthesis.

Strong evidence against temperature fluctuations as the cause of the dichotomy between ORLs and CELs is provided by Infrared Space Observatory (ISO) measurements of mid- and 

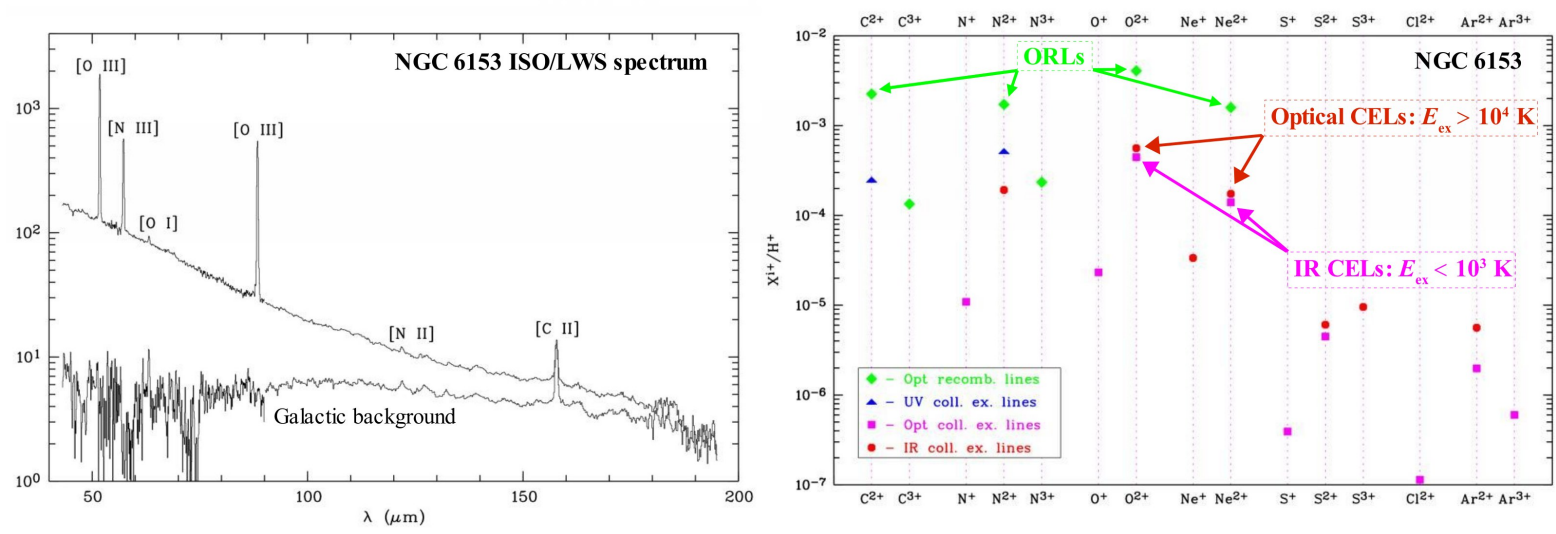

Figure 1.5: Left: The far-IR spectrum of NGC 6153; Right: Comparisons of ionic abundances of NGC 6153 deduced from ORLs, and from UV, optical and IR CELs. (Adapted from Liu et al., 2000)

far-IR fine-structure lines, such as the [Ne III] 15.5- $\mu \mathrm{m}$ and [O III] 52- and 88- $\mu \mathrm{m}$ (Liu et al., 2001a). Although collisionally excited, IR fine-structure lines have, unlike their optical and UV counterparts, excitation energies less than $\sim 1,000 \mathrm{~K}$ (Fig. 1.2), and are therefore insensitive to temperature or temperature fluctuations. If temperature fluctuations are indeed at work, leading to an overestimated $T_{\mathrm{e}}\left(\left[\begin{array}{ll}\mathrm{O} & \mathrm{III}\end{array}\right]\right)$ and consequently an underestimated $\lambda \lambda 4959,5007 \mathrm{O}^{++} / \mathrm{H}^{+}$abundance, then one expects a higher abundance from the 52- and 88- $\mu \mathrm{m}$ fine-structure lines comparable to the value yielded by $\mathrm{O}$ II ORLs. ISO measurements and subsequent analyses reveal, however, otherwise. Fig. 1.5 shows that in the case of NGC 6153, all CELs - UV, optical and IR, regardless of their excitation energy and critical density, yield ionic abundances that are consistently a factor of $\sim 10$ lower than ORLs.

To account for the multi-waveband observations of NGC6153, Liu et al. (2000) postulated that the nebula contains a previously unknown component of high-metallicity gas, presumably in the form of H-deficient inclusions embedded in the diffuse nebula of "normal" (i.e. about solar) composition. Due to the efficient cooling of abundant metals, this H-deficient gas has an electron temperature of only $\sim 1,000 \mathrm{~K}$, too low to excite any optical or UV CELs (thus invisible via the latter). Yet, the high metallicity combined with a very low electron temperature make those H-deficient inclusions powerful emitters of heavy element ORLs. In this picture, ORLs and CELs yield discrepant electron temperatures and ionic abundances because they probe two different gas components that co-exist in the same 
Table 1.1: Comparison of $T_{\mathrm{e}}$ 's deduced from CELs and from ORLs/continua ${ }^{a}$

\begin{tabular}{lccccc}
\hline Nebula & $\operatorname{adf}\left(\mathrm{O}^{++} / \mathrm{H}^{+}\right)$ & $\begin{array}{c}T_{\mathrm{e}}([\mathrm{O} \text { III }] \\
(\mathrm{K})\end{array}$ & $\begin{array}{c}T_{\mathrm{e}}(\mathrm{BJ}) \\
(\mathrm{K})\end{array}$ & $\begin{array}{c}T_{\mathrm{e}}(\mathrm{He} \mathrm{I}) \\
(\mathrm{K})\end{array}$ & $\begin{array}{c}T_{\mathrm{e}}(\mathrm{O} \text { II }) \\
(\mathrm{K})\end{array}$ \\
\hline NGC 7009 & 4.7 & 9980 & 7200 & 5040 & 420 \\
H 1-41 & 5.1 & 9800 & 4500 & 2930 & $<288$ \\
NGC 2440 & 5.4 & 16150 & 14000 & & $<288$ \\
Vy 1-2 & 6.2 & 10400 & 6630 & 4430 & 3450 \\
IC 4699 & 6.2 & 11720 & 12000 & 2460 & $<288$ \\
NGC 6439 & 6.2 & 10360 & 9900 & 4900 & 851 \\
M 3-33 & 6.6 & 10380 & 5900 & 5020 & 1465 \\
M 2-36 & 6.9 & 8380 & 6000 & 2790 & 520 \\
IC 2003 & 7.3 & 12650 & 11000 & 5600 & $<288$ \\
NGC 6153 & 9.2 & 9120 & 6000 & 3350 & 350 \\
DdDm 1 & 11.8 & 12300 & 11400 & 3500 & \\
Vy 2-2 & 11.8 & 13910 & 9300 & 1890 & 1260 \\
NGC 2022 & 16.0 & 15000 & 13200 & 15900 & $<288$ \\
NGC 40 & 17.8 & 10600 & 7000 & 10240 & \\
M 1-42 & 22.0 & 9220 & 4000 & 2260 & $<288$ \\
NGC 1501 & 31.7 & 11100 & 9400 & & \\
Hf 2-2 & 71.2 & 8820 & 900 & 940 & 630 \\
\hline
\end{tabular}

${ }^{a}$ Adapted from Liu (2006a). $T_{\mathrm{e}}$ (He I)'s were derived from the He I $\lambda 5876 / \lambda 4472$ and $\lambda 6678 / \lambda 4472$ line ratios and were typically accurate to 20 per cent (c.f. Zhang et al., $2005)$. Values of $T_{\mathrm{e}}(\mathrm{O}$ II $)$ were deduced from the $\mathrm{O}$ II $\lambda 4089 / \lambda 4649$ line ratio and had typical uncertainties of 30 per cent.

nebula but have vastly different physical and chemical characteristics. Empirical analysis of Liu et al. (2000) as well as follow-up 1D photoionization modeling (Péquignot et al., 2002) shows that a small amount of H-deficient material, about one Jupiter mass, is sufficient to account for the strengths of heavy element ORLs observed in NGC 6153.

The increasingly lower values of Balmer jump temperature $T_{\mathrm{e}}(\mathrm{BJ})$ found for nebulae of larger and larger adf's: 6,000 K in NGC6153 (adf = 9.2), 4,000 K in M 1-42 (adf = 22) and $900 \mathrm{~K}$ in Hf 2-2 ( $\mathrm{adf}=71)$, provide the smoking gun evidence that nebulae contain two regimes of vastly different physical properties. Further evidence is provided by careful analyses of the He I and heavy element recombination line spectra, which show that the average emission temperatures of the He I and O II ORLs are even lower than indicated by the $\mathrm{H}$ I Balmer discontinuity (Liu 2003). In general, it is found that, $T_{\mathrm{e}}(\mathrm{O}$ II $) \leq T_{\mathrm{e}}(\mathrm{He} \mathrm{I}) \leq$ $T_{\mathrm{e}}(\mathrm{BJ}) \leq T_{\mathrm{e}}([\mathrm{O} \mathrm{III}])$ (Table1.1; c.f. Liu, 2006a and references therein), as one expects in the dual-abundance scenario proposed by Liu et al. (2000). 
Detailed 3D photoionization models of NGC 6153 with and without H-deficient inclusions have been constructed by Yuan et al. [2010, in preparation; c.f. Figs. 1.6a) and b)] using MOCASSIN, a Monte Carlo photoionization code capable of dealing with nebulae of arbitrary geometry and composition (Ercolano et al. 2003a). In their models, the main nebula was modeled with a chemically homogeneous ellipsoid of "normal composition" (i.e. about solar as yielded by the CEL analysis). To mimic the bipolar shape of the nebula, the density in the ellipsoid was allowed to decrease from the equator to the poles. In addition, to reproduce the strengths of low-ionization lines, such as [C I] $\lambda \lambda 9824,9850,[\mathrm{~N}$ I] $\lambda \lambda 5198,5200$, [N II] $\lambda \lambda 6548,6584$, [O I] $\lambda \lambda 6300,6363$ and [O II] $\lambda \lambda 3727,3729$, an equatorial ring of the same chemical composition but of a higher density was added. The presence of a high-density torus is supported by a high resolution spectrum obtained with the Manchester Echelle Spectrograph mounted on the Anglo-Australian Telescope. The spectrum, centered on $\mathrm{H} \alpha$ and the $[\mathrm{N}$ II] $\lambda \lambda 6548,6584$ lines and obtained with a long-slit oriented in PA $=123$ deg and through the central star, revealed two high velocity emission spots at the positions of the bright shell, one on each side of the central star. The spots are particularly bright in [N II] and have respectively blue- and red-shifted velocities relative to the $\mathrm{H} \alpha$ emission from the nearby nebular shell. The model fits the CEL measurements well except for a few lines [c.f. the top panel of Fig. 1.6b)]. The model still underestimates the [C I] $\lambda 9850$ line by a factor of three even after the introduction of the high-density torus. The model also underestimates, by about a factor of two, the fluxes of the $\mathrm{N}$ III] $\lambda 1744$ and [Ar III] 8.9- and 21.8- $\mu$ m lines. The latter three lines were however only marginally detected, by the IUE and ISO, respectively, and the discrepancies may well be due to measurement errors. The largest discrepancy is found for the [Ne II] $12.8-\mu \mathrm{m}$ line where the model underestimates the observation by more than a factor of five.

The chemically homogeneous model however fails to reproduce the strengths of all heavy element ORLs by a factor of ten. To account for them, metal-rich inclusions were added, in the form of clumps of H-deficient material. The clumps were distributed spherically symmetrically with a radial number density profile that reproduced the $\mathrm{O}$ II and $\mathrm{C}$ II ORL surface brightness distributions deduced from the long-slit observations (Liu et al., 2000). Given that the H-deficient inclusions have not been resolved, even with the HST/STIS spectroscopy with a slit width of 0.2 arcsec (Yuan et al., 2010), they must have dimensions smaller 

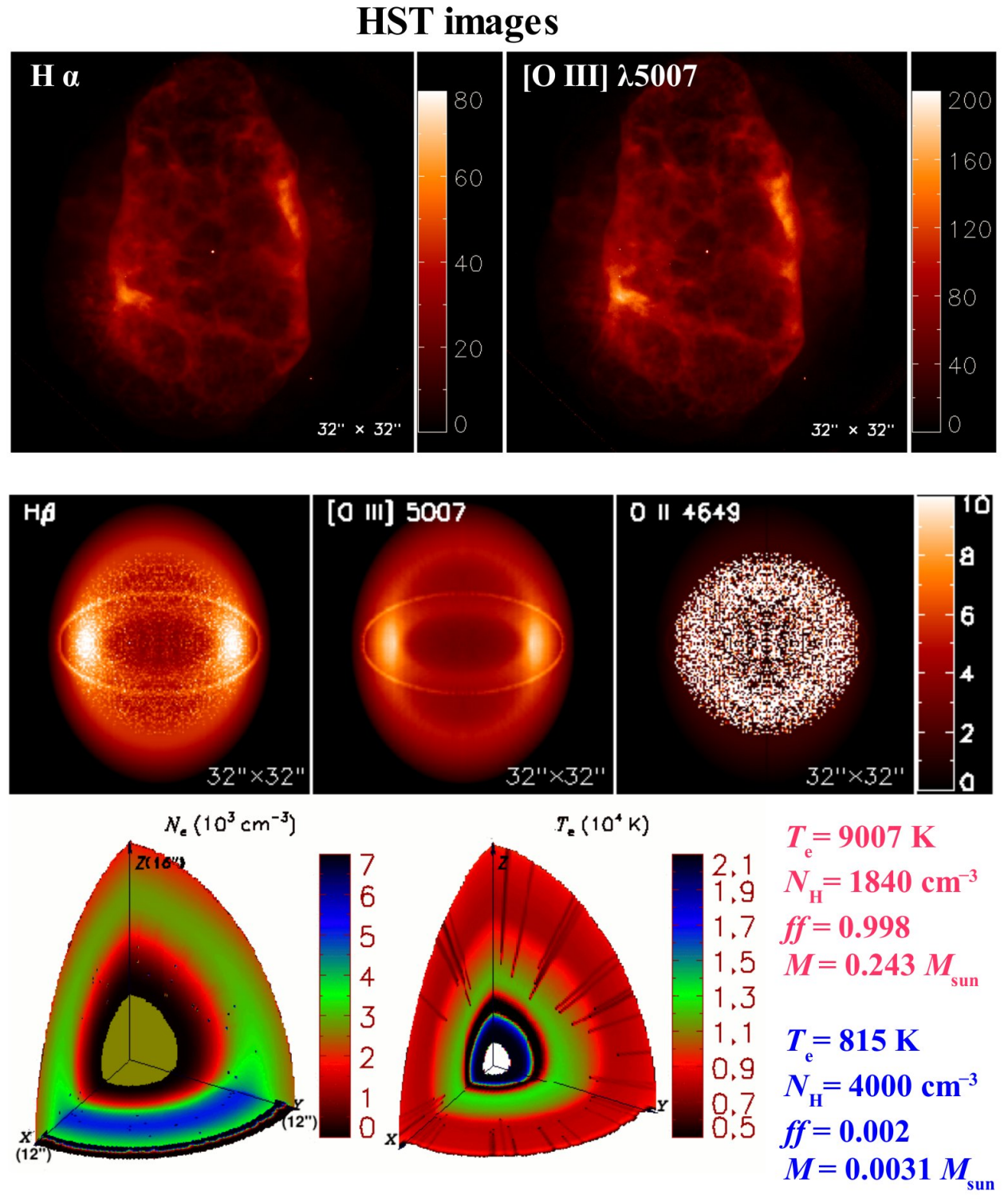

Figure 1.6: a) From top to bottom: HST monochromatic images of NGC 6153 in $\mathrm{H} \alpha$ and [O III] $\lambda 5007$ (north is up and east to the left); monochromatic images predicted by the best-fit model incorporating H-deficient inclusions; and the model distributions of electron density and temperature. 

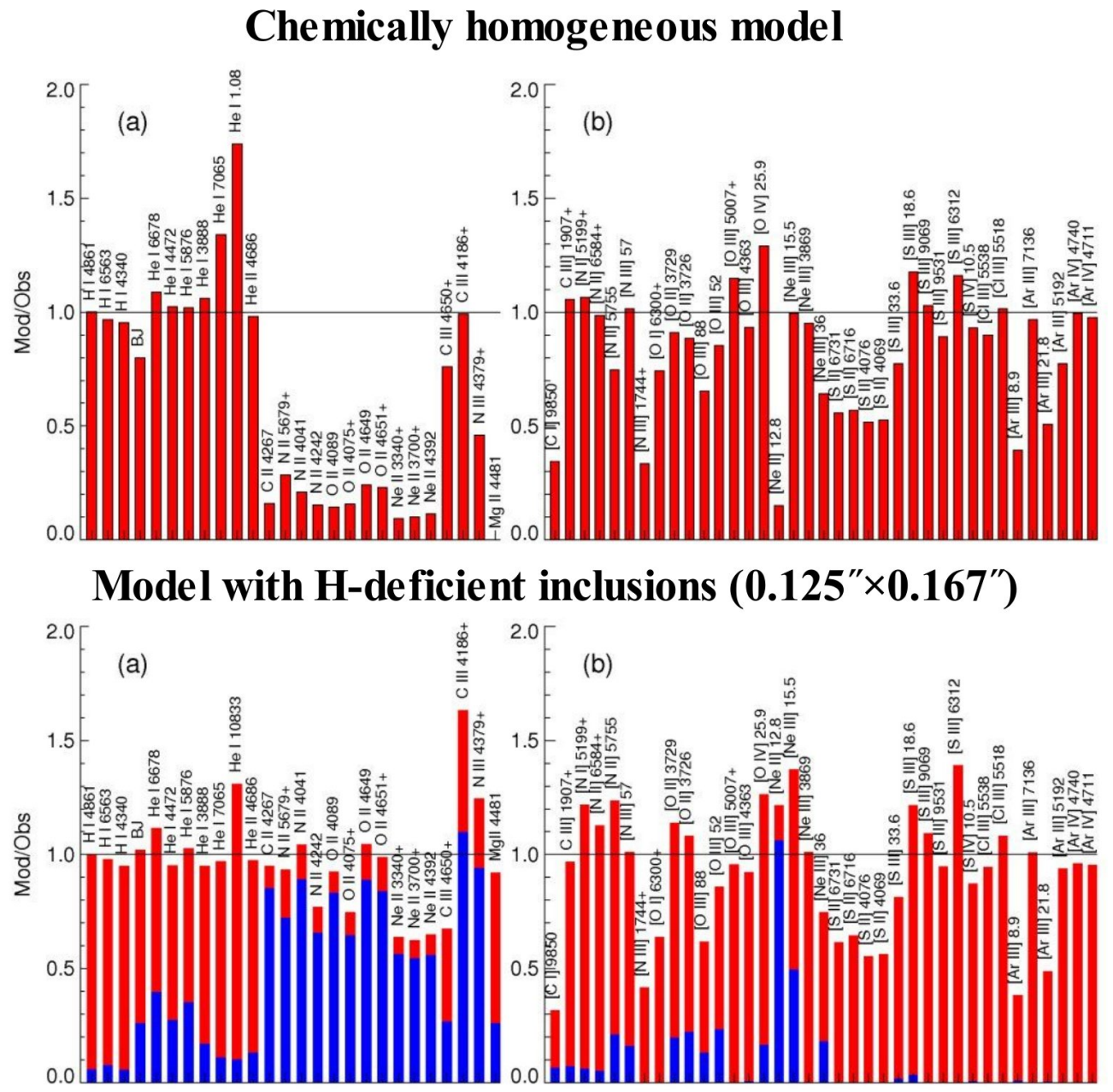

Normal component

H: 10000 He: 1000 C: 3.20

$\mathrm{N}: 3.80 \quad 0: 5.53 \quad \mathrm{Ne}: 1.76$
H-deficient component

H: 10000 He: 5000 C: 177

N: $150 \quad$ O: $440 \quad$ Ne: 177

H-deficient knots are cooled by infrared fine-structure lines: [O III] $52 \mu \mathrm{m}$, [Ne II] $12.8 \mu \mathrm{m}$ and [Ne III] $15.5 \mu \mathrm{m}$

Figure 1.6: - continued. b) Comparison of model line intensities with observations for the best-fit chemically homogeneous model (top) and model containing H-deficient inclusions (bottom). In the bottom panel, blue and red color bars denote, respectively, contributions of line fluxes from the cold, H-deficient inclusions and from the hot, diffuse gas of "normal composition". 
than $\sim 0.2 \mathrm{arcsec}$, or $300 \mathrm{AU}$ at the assumed distance of $1.5 \mathrm{kpc}$ of NGC 6153 . The smallest size of inclusions that can be modeled with the MOCASSIN was limited by the memory available per processor of the computer employed. For NGC 6153, a grid of $48^{3}$ cells, each of $1 / 4 \operatorname{arcsec}$ by $1 / 3$ arcsec, was used to model $1 / 8$ of the nebula using a SGI Altix 330 cluster of 8 dual-core processors and $8 \mathrm{~Gb}$ memory per processor. Models that doubled the resolution were also constructed using the NASA/Ames Columbia Supercomputer. Results from one of these high resolution models are shown in Fig.1.6a) and b). The best-fit dual-abundance model incorporating $\mathrm{H}$-deficient inclusions matches all available observations reasonably well and successfully reproduces strengths of heavy element ORLs. With a total mass of only one Jupiter mass, or just over $1 \%$ that of the whole nebula, a helium abundance 5 times and CNONe abundances 40 - 100 times higher than the main diffuse nebula of "normal composition", the H-deficient inclusions account for approximately, 5, 35 and 100\% of the observed fluxes of H I, He I and heavy element ORLs, respectively, but produce essentially nil optical and UV CEL emission. Cooled to about $800 \mathrm{~K}$ by IR fine-structure lines of heavy elemental ions, notably the [Ne II] $12.8-\mu \mathrm{m}$, [Ne III] $15.5-\mu \mathrm{m}$ and [O III] $52-\mu \mathrm{m}$ lines, the inclusions are important contributors of those lines, so for the $\mathrm{H}$ I recombination continuum Balmer discontinuity (about 30\%). The inclusions even dominate the [Ne II] 12.8- $\mu \mathrm{m}$ line flux. Note that gas densities in the H-deficient inclusions are not well constrained at the moment, partly due to the lack of suitable atomic data and diagnostic tools (see below, §1.8). This leads to some uncertainties in the deduced total mass of the H-deficient material. In the current treatment, the inclusions have densities and temperatures that are roughly in pressure equilibrium with the surrounding diffuse medium of higher temperatures and lower densities. Physically, one envisions individual inclusions to be optically thick such that they can survive long enough to have observational effects. Proper modeling of optically thick knots will however require a separate set of fine grid for each of them to resolve the ionization and thermal structures, and thus demand even more computing resources.

The average elemental abundances for the whole nebula of NGC 6153, including the diffuse nebula as well as H-deficient inclusions embedded in it, are $0.102,3.9 \times 10^{-4}, 4.4 \times 10^{-4}$, $7.3 \times 10^{-4}$ and $2.4 \times 10^{-4}$ for He, C, N, O and Ne, respectively. Except for helium, elemental abundances of the "normal" component are close to those deduced from the empirical method based on CELs (Liu et al., 2000), and are about 30 per cent lower than the average 
abundances for the whole nebula. For helium, the abundance of the "normal" component is about 40 per cent lower than derived from the empirical method based on the observed strengths of helium ORLs, and actually comes closer to the average helium abundance of the whole nebula. That NGC 6153 has a lower helium abundance than implied by the empirical analysis is supported by a measurement of the near IR He I $\lambda 10830$ line, for which excitation from the $2 \mathrm{~s}^{3} \mathrm{~S}$ meta-stable level by electron impacts dominates the emission (Yuan et al., 2010).

Helium lines observable in the optical and UV are all dominated by recombination excitation, and their strengths can be strongly enhanced by a small amount of H-deficient, ultra-cold plasma posited in the nebula. If H-deficient inclusions are indeed fully responsible for the ORL versus CEL dichotomy ubiquitously found in PNe and H II regions, then their presence may have a profound consequence on the helium abundances deduced for those objects, as well as on the primordial helium abundance determined by extrapolating the helium abundances of metal-poor H II galaxies to zero metallicity - the inferred primordial helium abundances from standard analyses would be too high (Zhang et al., 2004).

Corroborative evidence that ORLs arise from distinct regions of very low temperature plasma is provided by analyses of emission line profiles. Spectroscopy at a resolution of $6 \mathrm{~km} \mathrm{~s}^{-1}$ of NGC 7009 shows that O II ORLs are significantly narrower than the [O III] forbidden lines (Liu, 2006b). Observations at an even higher resolution of $2 \mathrm{~km} \mathrm{~s}^{-1}$ of NGC 7009 and NGC 6153 yield complex multi-velocity-components and differing line profiles for the O II ORLs and for the [O III] forbidden lines. The profiles of the [O III] $\lambda 5007$ nebular and $\lambda 4363$ auroral lines also differ significantly. These results suggest that ionized regions of vastly different temperatures (and radial and thermal velocities) co-exist in those nebulae (Barlow et al., 2006). Zhang (2008) shows that the differences in the observed line profiles of the O II ORLs and the [O III] CELs are hard to explain in a chemically homogeneous nebula. 


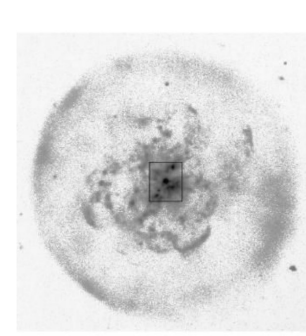

Abell 30
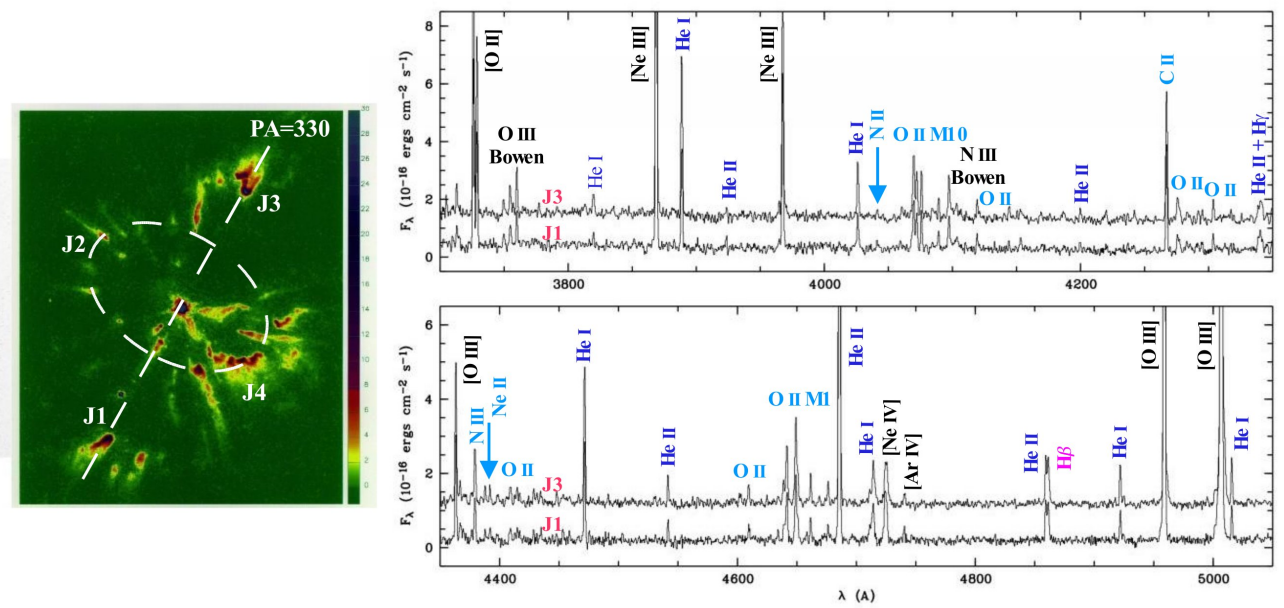

Figure 1.7: Left: The optical images of Abell 30, showing the H-deficient knots near the nebular center (adapted from Borkowski et al., 1993); Right: The optical spectrum of the polar knots J 1 and J 3 showing prominent ORLs of C, N, O and Ne ions (adapted from Wesson et al., 2003). Note the faintness of $\mathrm{H} \beta$ at $4861 \AA$.

\subsection{Spectroscopy of PNe harboring $\mathrm{H}$-deficient inclusions}

H-deficient clumps were previously known to exist in a rare class of old PNe, including Abell 30, 58 and 78, IRAS 15154-5258 and 18333-2357 (e.g. Harrington, 1996). They are identified as PNe that experience a last helium shell flash that brings them back to the AGB to repeat the PN evolution stage, the so-called "born-again" PNe (Iben et al., 1983). In Abell 30, HST imaging reveals a host of knots embedded near the center of a round, limbbrightened faint nebula of angular diameter $127 \operatorname{arcsec}$ (Borkowski et al., 1993; c.f. Fig. 1.7). They include two point-symmetric polar knots along $\mathrm{PA}=331^{\circ}$ at angular distances 6.66 and 7.44 arcsec from the central star, plus a number of knots loosely delineating an equatorial disk or ring. Deep optical spectra of knots J 1 and J 3 were obtained by Wesson et al. (2003). The spectra show prominent recombination lines from CNONe ions, remarkably similar to those seen in other PNe of large adf's, such as Hf 2-2 in Fig.1.4, except that in Abell 30 the strengths of those CNONe ORLs relative to $\mathrm{H} \beta$ are much higher. Detailed ORL analyses show that the ORLs are indeed emitted under very low temperatures of only a few hundred Kevin. The result is corroborated by detailed 3D photoionization modeling (Ercolano et al., 2003b) as well as by a similar analysis of the H-deficient knot found at the center of Abell 58 by Wesson et al. (2008). 


\subsection{Origins of $\mathrm{H}$-deficient inclusions}

The origin of H-deficient material is not well understood and their existence in PNe, and possibly also in H II regions (see, for example, Esteban et al. 2002 and Tsamis et al. 2003a), is not predicted by the current theory of stellar evolution. For PNe harboring H-deficient central stars, such as the Wolf-Rayet and PG 1159 stars, while the scenario of a single postAGB star experiencing a last helium shell flash seems to be able to match the photospheric abundances of the star, not all PNe exhibiting large adf's have an H-deficient central star (e.g. NGC 7009). The process and mechanism by which H-deficient knots might be ejected in such systems are not understood. More importantly, detailed ORL abundance analyses by Wesson et al. $(2003,2008)$ show that the H-deficient knots found in Abell 30 and Abell 58 are oxygen-rich, not carbon-rich as one expects in the scenario of "born-again" PNe.

The precise collinearity of the two polar knots in Abell 30 with the central star, to within 5 arcmin, is hard to explain by single star evolution, and suggests instead the action of a bipolar jet from, for example, an accretion disk in a binary system (Harrington, 1996; De Marco, 2008). In this respect, it is interesting to note that Abell 58 is known to have experienced a nova-like outburst in 1917 (Clayton and De Marco, 1997). Hf 2-2 has also been found to be a close binary system with a period of $0.398571 \mathrm{~d}$ (Lutz et al., 1998). By comparing the known properties of Abell 58 with those of Abell30, Sakurai's Object and several novae and nova remnants, Wesson et al. (2008) argue that the elemental abundances in the H-deficient knots of Abell 30 and Abell 58 have more in common with neon novae than with Sakurai's Object, which is believed to have undergone a final helium flash.

An alternative to the scenario of H-deficient inclusions being ejecta of nucleo-processed material is that they derive from metal-rich planetary material, such as icy planetesimals, that once orbited the PN progenitor star. As the star enters the PN phase by ejecting the envelope and evolves to become a luminous, hot white dwarf, the strong stellar winds and UV radiation fields begin to photoionize and strip gas off the planetesimals, now embedded in the PN (Liu, 2003, 2006a). As analyses show that only a few Jupiter masses metal-rich material is required to explain the observed strengths of ORLs, the idea may not be so eccentric as it first looks. Evaporating proto-planetary disks around newly formed stars 
(such as the proplyds found in the Orion Nebula) may likewise provide a natural solution to the problem of ORL versus CEL dichotomy similarly found in H II regions.

\subsection{The need for new atomic data}

Atomic data relevant for the study of emission line nebulae, including collision strengths and effective recombination coefficients, are generally calculated for a temperature range $5,000-20,000 \mathrm{~K}$, typical for photoionized nebulae of solar composition. The finding that heavy element ORLs emitted by the H-deficient knots in the "born-again" PNe Abell 30 and Abell 58 arise from ultra-cold plasma of only a few hundred Kevin, as well as the compelling evidence accumulated so far that points to the presence of similar cold H-deficient inclusions in other PNe, call for new atomic data, in particular the effective recombination coefficients applicable in such low temperature environments. New plasma diagnostics that use only ORLs, not CELs, need to be developed so that physical and chemical properties of the Hdeficient knots/inclusions, such as temperature, density, elemental abundances and mass, can be determined reliably, a prerequisite to unravel their origins.

The ratios of the intensities of ORLs from states of different orbital angular momenta show some temperature dependence and thus can be used to measure the average temperature under which the lines are emitted (Liu, 2003). In addition, as pointed out by Liu (2003), the dependence on density of the relative populations of the fine-structure levels of the ground spectral term of the recombining ions, such as $\mathrm{O}^{2+} 2 \mathrm{p}^{2}{ }^{3} \mathrm{P}_{0,1,2}$, leads to variations of the relative intensities of recombination lines as a function of density. In the case of O II, for example, lines from high- $l$ states, such as the $3 \mathrm{p}^{4} \mathrm{D}_{7 / 2}^{\mathrm{o}}-3 \mathrm{~s}^{4} \mathrm{P}_{5 / 2} \lambda 4649$ transition (c.f. Fig. (1.2) weakens as density decreases due to the underpopulation of the $\mathrm{O}^{2+} 2 \mathrm{p}^{2}{ }^{3} \mathrm{P}_{2}$ level under low densities. This effect opens up the possibility of determining electron density using ORLs.

New effective recombination coefficients, calculated down to temperatures of $\sim 100 \mathrm{~K}$ and taking into account the dependence on density of level populations of the ground states of the recombining ion, have now been carried out for the O II (Bastin and Storey, 2006) and N II 

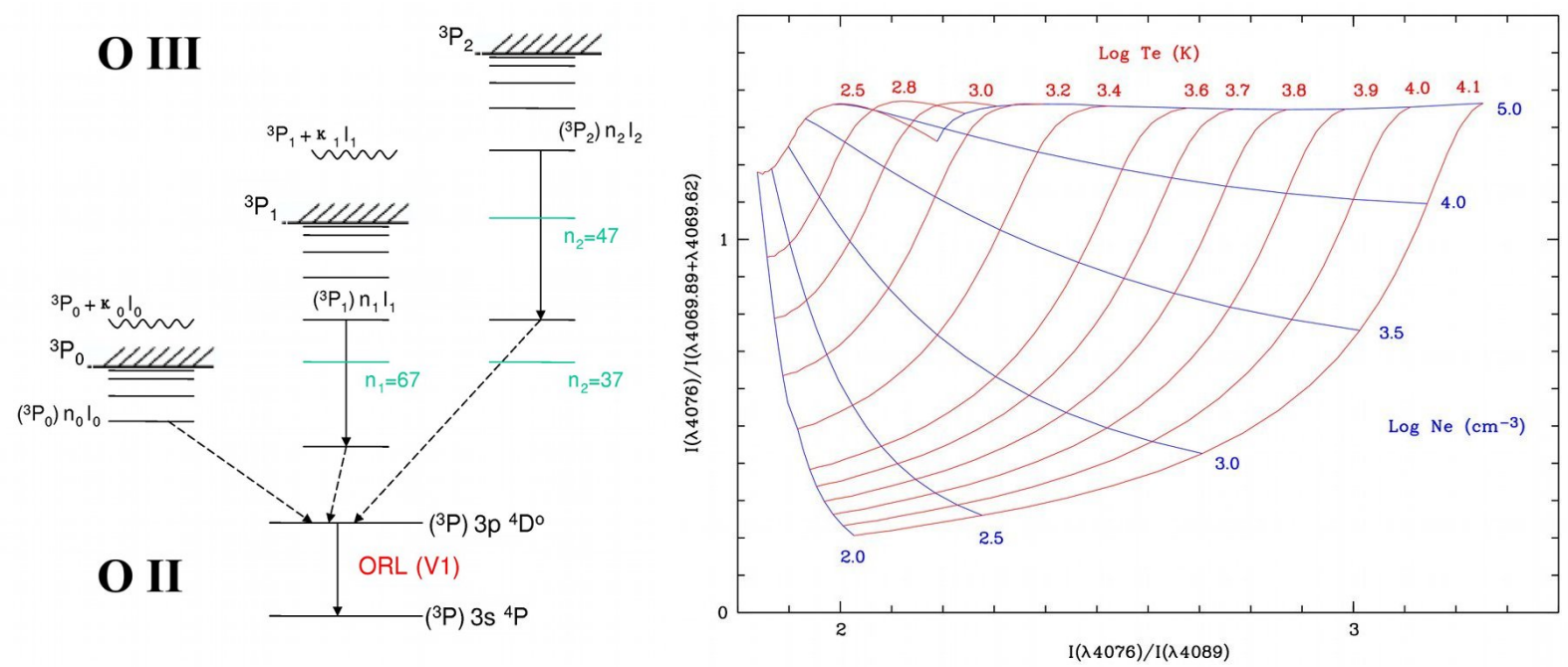

Figure 1.8: Left: Schematic diagram illustrating the low temperature di-electronic recombination of $\mathrm{O}$ II via the low-lying fine-structure auto-ionizing states. Note that for $n_{1} \geq 67$ and $n_{2} \geq 37$, the doubly-excited $\mathrm{O}$ II $2 \mathrm{p}^{2}\left({ }^{3} \mathrm{P}_{1}\right) n_{1} l_{1}$ and $2 \mathrm{p}^{2}\left({ }^{3} \mathrm{P}_{2}\right) n_{2} l_{2}$ states fall above the $\mathrm{O}$ III ground level $2 \mathrm{p}^{2}{ }^{3} \mathrm{P}_{0}$. Similarly, for $n_{2} \geq 47$, O II $2 \mathrm{p}^{2}\left({ }^{3} \mathrm{P}_{2}\right) n_{2} l_{2}$ states have energies above the $\mathrm{O}$ III first excited fine-structure level $2 \mathrm{p}^{2} \mathrm{P}_{1}$. For temperatures of a few hundred Kelvin, di-electronic recombination via those low-lying fine-structure auto-ionizing states becomes an important process; Right: Loci of the O II recombination line ratios $\lambda 4076 / \lambda 4089$ and $\lambda 4076 / \lambda 4069$ for different $T_{\mathrm{e}}$ 's and $N_{\mathrm{e}}$ 's (based on data provided by Dr. P. J. Storey).

(Fang, Storey and Liu, in preparation) recombination spectra. Fig. 1.8 plots loci of the O II $I(\lambda 4076) / I(\lambda 4089)$ and $I(\lambda 4076) / I(\lambda 4070)$ intensity ratios for different temperatures and densities. The diagram also illustrates the importance of di-electronic recombination via the low-lying fine-structure auto-ionizing states under very low temperatures. Preliminary applications of these new atomic data and diagnostics to observations yield lower temperatures and higher densities than CELs, as one expects.

\subsection{Summary}

To summarize, nearly one and half centuries after William Huggins' discovery of bright emission lines in the spectra of gaseous nebulae, enormous progress has been achieved in understanding them. In particular, we believe we now understand the faint ORLs emitted by heavy element ions, more than half a century after Wyse's pioneer work on O II. The theory of photoionized gaseous nebulae seems to be solid. Yet any progress in our understanding 
of their nature seems to be always accompanied by the emergence of new puzzles. Clearly, those beautiful heavenly objects, queens of the night, are more delicate than we think and still hold some fascinating secrets from us. With dedicated observations, more discoveries are sure to come. 


\section{References}

[1] Aller, L. H. and Menzel, D. H. (1945). Physical Processes in Gaseous Nebulae. XVIII. The chemical composition of the planetary nebulae. ApJ, 102, 239

[2] Aller, L. H., Baker, J. G. and Menzel D. H. (1939). Physical processes in gaseous nebulae. VIII. The ultraviolet radiation field and electron temperature of an optically thick nebula. ApJ, 90, 601

[3] Baker, J. G. and Menzel, D. H. (1938). Physical processes in gaseous nebulae. III. The Balmer decrement. ApJ, 88, 52;

[4] Baker, J. G., Menzel, D. H. and Aller, L. H. (1938). Physical processes in gaseous nebulae. V. Electron temperatures. ApJ, 88, 422

[5] Barker, T. (1978). Spectrophotometry of planetary nebulae. I - Physical conditions. ApJ, 219, 914

[6] Barker, T. (1991). The ionization structure of planetary nebulae. X - NGC 2392. ApJ, 371,217

[7] Barlow, M. J., Liu, X.-W., Péquignot, D., Storey, P. J., Tsamis, Y., Morisset, C., (2003). $\mathrm{PN}$ recombination line abundances for magnesium, silicon and sulphur. In Planetary Nebulae: Their Evolution and Role in the Universe, Proc. IAU Symp. \#209. Eds. S. Kwok, M. Dopita and R. Sutherland. PASP. pp.373-374

[8] Barlow, M. J., Hales, A. S., Storey, P. J., Liu, X.-W., Tsamis, Y. G. and Aderin, M. E. (2006). bHROS high spectral resolution observations of PN forbidden and recombination line profiles planetary nebulae. In Planetary Nebulae in our Galaxy and Beyond, Proceedings of the IAU Symposium \#234. Eds. M. J. Barlow and R. H. Méndez Cambridge: Cambridge University Press. p.367

[9] Bastin, R. J. and Storey, P. J. (2006). Recombination line spectroscopy: the O II spectrum. In Planetary Nebulae in our Galaxy and Beyond, Proceedings of the IAU Symposium \#234. Eds. M. J. Barlow and R. H. Méndez. Cambridge: Cambridge University Press. p.369

[10] Borkowski, K. J., Harrington, J. P., Tsvetanov, Z., Clegg, R. E. S. (1993). HST imaging of hydrogen-poor ejecta in Abell 30 and Abell 78 - Wind-blown cometary structures. ApJ, 415, 47

[11] Bowen, I. S. (1927a). Series spectra of boron, carbon, nitrogen, oxygen and fluorine. 
Phys.Rev., 29, 231.

[12] Bowen, I. S. (1927b). The Origin of the Nebulium Spectrum. Nature, 120, 473

[13] Bowen, I. S. (1927c). The origin of the chief nebular lines. PASP, 39, 295

[14] Bowen, I. S. (1934). The Excitation of the Permitted O III Nebular Lines. PASP, 46, 146

[15] Bowen, I. S. (1935). The Spectrum and Composition of the Gaseous Nebulae. ApJ, 81, 1

[16] Bowen, I. S. (1938). The image-slicer, a device for reducing loss of light at slit of stellar spectrograph. ApJ, 88, 113

[17] Bowen, I. S. and Wyse, A. (1939). The spectra and chemical composition of the gaseous nebulae, NGC 6572, 7027, 7662. Lick Obs. Bull., 19, 1

[18] Burgess, A. (1958). The hydrogen recombination spectrum. MNRAS, 118, 477

[19] Clayton, G. C. and De Marco, O. (1997). The evolution of the final helium shell flash star V 605 Aquilae from 1917 to 1997. AJ, 114, 2679

[20] Cunto, W., Mendoza, C., Ochsenbein, F. and Zeippen, C. J. (1993). Topbase at the CDS. A\&A, 275, 5

[21] De Marco, O. (2008). [WC] and PG 1159 central stars of planetary nebulae: the need for an alternative to the born-again scenario. In Hydrogen-Deficient Stars. Eds. K. Werner and T. Rauch. PASP: San Francisco. p.209

[22] Ercolano, B., Barlow, M. J., Storey, P. J. and Liu, X.-W. (2003a). MOCASSIN: a fully three-dimensional Monte Carlo photoionization code. MNRAS, 340, 1136

[23] Ercolano, B., Barlow, M. J., Storey, P. J., Liu, X.-W., Rauch, T. and Werner K. (2003b). Three-dimensional photoionization modelling of the hydrogen-deficient knots in the planetary nebula Abell 30. MNRAS, 344, 1145

[24] Esteban, C., Peimbert, M., Torres-Peimbert, S. and Rodríguez, M. (2002). Optical recombination lines of heavy elements in giant extragalactic H II regions. ApJ, 581, 241

[25] Flower, D. R. (1969). The ionization structure of planetary nebulae - VII. The heavy elements. MNRAS, 146, 171; The ionization structure of planetary nebulae - VIII. Models of the nebulae NGC 7662 and IC 418. ibid, 146, 243

[26] Goodson, W. L. (1967). Ionisationsstruktur planetarischer nebel. Z.Astrophys., 66, 118

[27] Harrington, J. P. (1968). Ionization stratification and thermal stability in model plane- 
tary nebulae. ApJ, 152, 943

[28] Harrington, J. P. (1996). Observations and models of H-deficient planetary nebulae. In Hydrogen-Deficient Starsi, ASP Conf. Ser. Vol. 96. eds. C. S. Jeffery, U. Hebera. PASP: San Francisco. p.193

[29] Herschel, W. (1785), On the construction of the heavens. Phil.Trans.R.Soc.London, 75, 213.

[30] Hjellming, R. (1966). Physical processes in H II regions. ApJ, 143, 120

[31] Huggins, W. (1882). Note on the photographic spectrum of the Great Nebula in Orion. Proc. R. Soc. London, 33, 425

[32] Huggins, W. and Millar, W. A. (1864). On the spectra of some of the fixed stars. Phil. Trans. R. Soc. London, 154, 437

[33] Iben, I., Jr., Kaler, J. B., Truran, J. W. and Renzini, A. (1983). On the evolution of those nuclei of planetary nebulae that experience a final helium shell flash. ApJ, 264, 605

[34] Kaler, J. B. (1966). Hydrogen and Helium Spectra of Gaseous Nebulae. ApJ, 143, 722

[35] Kirchhoff, G. and Bunsen, R. W. (1860). Chmical analysis by obseration of spectra. Annalen der Physik und der Chemie (Poggendorff), Vol. 110, pp.161-189.

[36] Liu, X.-W. (2003). Probing the Dark Secrets of PNe with ORLs (invited review). In Planetary Nebulae: Their Evolution and Role in the Universe, Proc. IAU Symp. \#209. Eds. S. Kwok, M. Dopita and R. Sutherland. PASP. pp.339-346

[37] Liu, X.-W. (2006a). Optical recombination lines as probes of conditions in planetary nebulae. In Planetary Nebulae in our Galaxy and Beyond, Proceedings of the IAU Symposium \#234. Eds. M. J. Barlow and R. H. Méndez. Cambridge: Cambridge University Press. pp.219-226

[38] Liu, X.-W. (2006b). Plasma diagnostics and elemental abundance determinations for PNe current status. In Planetary nebulae beyond the Milky Way, eds. J. Walsh, L. Stanghellini and N. Douglas, p.169

[39] Liu, X.-W., Barlow, M. J., Zhang, Y., Bastin, R. J. and Storey, P. J. (2006). Chemical abundances for Hf 2-2, a planetary nebula with the strongest-known heavy-element recombination lines. MNRAS, 368, 1959

[40] Liu, X.-W., Barlow, M. J., Cohen, M., Danziger, I. J., Luo, S.-G., Baluteau, J. P., 
Cox, P., Emery, R. J., Lim, T. and Péquignot, D. (2001a). ISO LWS observations of planetary nebula fine-structure lines. MNRAS, 323, 343

[41] Liu, X.-W. and Danziger, I. J. (1993). Electron temperature determination from nebular continuum emission in planetary nebulae and the importance of temperature fluctuations. MNRAS, 263, 256

[42] Liu, X.-W., Luo, S.-G., Barlow, M. J., Danziger, I. J. and Storey, P. J. (2001b). Chemical abundances of planetary nebulae from optical recombination lines - III. The Galactic bulge PN M 1-42 and M 2-36

[43] Liu, X.-W., Storey, P. J., Barlow, M. J. and Clegg, R. E. S. (1995). The rich O II recombination spectrum of the planetary nebula NGC 7009: new observations and atomic data. MNRAS, 272, 369

[44] Liu, X.-W., Storey, P. J., Barlow, M. J., Danziger, I. J., Cohen, M. and Bryce, M. (2000). NGC 6153: a super-metal-rich planetary nebula? MNRAS, 312, 585

[45] Liu, Y., Liu, X.-W., Luo, S.-G. and Barlow, M. J. (2004a). Chemical abundances of planetary nebulae from optical recombination lines - I. Observations and plasma diagnostics. MNRAS, 353, 1231

[46] Liu, Y., Liu, X.-W., Barlow, M. J. and Luo, S.-G. (2004b). Chemical abundances of planetary nebulae from optical recombination lines - II. Abundances derived from collisionally excited lines and optical recombination lines. MNRAS, 353, 1251

[47] Luo, S.-G., Liu, X.-W. and Barlow, M. J. (2001). Chemical abundances of planetary nebulae from optical recombination lines - II. The neon abundance of NGC 7009. MNRAS, 326, 1049

[48] Lutz, J., Alves, D. and Becker, A., et al. (1998). V and R magnitudes for planetary nebula central stars in the MACHO project galactic bulge fields. AAS, 192, 5309

[49] Mathis, J. S. and Liu, X-W. (1999). Observations of the [O III] $\lambda 4931 / \lambda$ lambda4959 line ratio and $\mathrm{O}^{+2}$ abundances in ionized nebulae. ApJ, 521, 212

[50] Menzel, D. H. and Aller, L. H. (1941). Physical processes in gaseous nebulae. XVI. The abundance of O III. ApJ, 94, 30

[51] Menzel, D. H., Aller, L. H. and Hebb, M. H. (1941). Physical processes in gaseous nebulae. XIII. ApJ, 93, 230.

[52] Miller, J. S. (1971). Photoelectric measurements of high-n Balmer lines in NGC 7027 
and NGC 7662. ApJ, 165, L101

[53] Miller, J. S. and Mathews, W. G. (1972). The recombination spectrum of the planetary nebula NGC 7027. ApJ, 172, 593

[54] Osterbrock, D. E. (1974). Astrophysics of Gaseous Nebulae. Sausalito: University Science Books.

[55] Osterbrock, D. E. and Ferland, G. J. (2005). Astrophysics of Gaseous Nebulae and Active Galactic Nuclei. Sausalito: University Science Books.

[56] Pagel, B. E. J. (1997). Nucleosynthesis and Chemical Evolution of Galaxies. Cambridge: Cambridge University Press

[57] Peimbert, M. (1967). Temperature determinations of H II regions. ApJ, 150, 825

[58] Peimbert, M. (1971). Planetary nebulae II. Electron temperatures and electron densities. Bol.Obs.Ton.Tac., 6, 29

[59] Péquignot, D., Amara, M., Liu, X.-W., Barlow, M. J., Storey, P. J., Morisset, C., TorresPeimbert, S. and Peimbert, M. (2002). Photoionization models for planetary nebulae with inhomogeneous chemical composition. RMxAC, 12, 142

[60] Rola, C. and Stasińska, G. (1994). The carbon abundance problem in planetary nebulae. A\&A, 282, 199

[61] Rubin, R. H. (1968). The Structure and Properties of H II Regions. ApJ, 153, 761

[62] Russell, H. N., Dugan, R. S., Stewart, J. Q. (1927). Astronomy. Boston: Ginn and Co.

[63] Schmidt B. (1932). Mitteilungen Hamburger Sternwarte in Bergedorf 7(36), 15. Reprinted: Selected Papers on Astronomical Optics, SPIE Milestone Ser. 73, 165 (1993)

[64] Seaton, M. J. (1954). Electron temperatures and electron densities in planetary nebulae. MNRAS, 114, 154

[65] Seaton, M. J. (1959). Radiative recombination of hydrogenic ions. MNRAS, 119, 81; The solution of capture-cascade equations for hydrogen. ibid, 119, 90

[66] Seaton, M. J. and Osterbrock, D. E. (1957). Relative [O II] intensities in gaseous nebulae. ApJ, 125, 66

[67] Seaton, M. J. (1960). H I, He I, and HeII intensities in planetary nebulae. MNRAS, 120, 326

[68] Seaton, M. J. (1987). Atomic data for opacity calculations: I. General description. J.Phys.B, 20, 6363 
[69] Shklovsky I. S. (1956). Astron. Zh., 33, 315

[70] Spitzer, L. (1948). The temperature of interstellar matter. I. ApJ, 107, 6

[71] Strömgren, B. (1939). The physical state of interstellar hydrogen. ApJ, 89, 526

[72] Tsamis, Y. G., Barlow, M. J., Liu, X.-W., Danziger, I. J. and Storey, P. J. (2003a). Heavy elements in Galactic and Magellanic Cloud H II regions: recombination-line versus forbidden-line abundances. MNRAS, 338, 687

[73] Tsamis, Y. G., Barlow, M. J., Liu, X.-W., Danziger, I. J. and Storey, P. J. (2003b). A deep survey of heavy element lines in planetary nebulae - I. Observations and forbiddenline densities, temperatures and abundances. MNRAS, 345, 186

[74] Tsamis, Y. G., Barlow, M. J., Liu, X.-W., Storey, P. J. and Danziger, I. J. (2004). A deep survey of heavy element lines in planetary nebulae - II. Recombination-line abundances and evidence for cold plasma. MNRAS, 353, 953

[75] Wang, W. and Liu, X.-W. (2007). Elemental abundances of Galactic bulge planetary nebulae from optical recombination lines. MNRAS, 381, 669

[76] Wesson, R., Barlow, M. J., Liu, X.-W., Storey, P. J., Ercolano, B. and de Marco, O. (2008). The hydrogen-deficient knot of the 'born-again' planetary nebula Abell 58 (V605 Aql). MNRAS, 383, 1639

[77] Wesson, R., Liu, X.-W. and Barlow, M. J. (2003). Physical conditions in the planetary nebula Abell 30. MNRAS, 340, 253

[78] Wesson, R., Liu, X.-W. and Barlow, M. J. (2005). The abundance discrepancy - recombination line versus forbidden line abundances for a northern sample of galactic planetary nebulae MNRAS, 362, 424

[79] Wood R. (1910). The echellette grating for the infra-red. Philos. Mag. 20 (series 6), 770-778

[80] Wright W. H. (1918). The wave lengths of the nebular lines and general observations of the spectra of the gaseous nebulae. Publ. Lick Obs., 13, 191

[81] Wyse, A. B. (1942). The Spectra of Ten Gaseous Nebulae. ApJ, 95, 356

[82] Zanstra, H. (1926). An application of the quantum theory to the luminosity of diffuse nebulae. Phys.Rev., 27, 644

[83] Zanstra, H. (1927). An application of the quantum theory to the luminosity of diffuse nebulae. ApJ, 65, 50 
[84] Zanstra, H. (1931). Luminosity of planetary nebulae and stellar temperatures. Publ.Dom.Astrophys.Obs. (Victoria), 4, 209

[85] Zhang, Y., Liu, X.-W., Wesson, R., Storey, P. J., Liu, Y. and Danziger, I. J. (2004). Electron temperatures and densities of planetary nebulae determined from the nebular hydrogen recombination spectrum and temperature and density variations. MNRAS, 351,935

[86] Zhang, Y., Liu, X.-W., Liu, Y. and Rubin, R. H. (2005). Helium recombination spectrum as temperature diagnostics for planetary nebulae MNRAS, 358, 457

[87] Zhang, Y. (2008). Emission line profiles as a probe of physical conditions in planetary nebulae. A\&A, 486, 221 


\section{Document Control Sheet-List of Elements in Chapter}

\section{Tables}

- Table 1:

- Title:

- Source/permission status/credit line:

- Table 2:

- Title:

- Source/permission status/credit line:

- Table 3:

- Title:

- Source/permission status/credit line:

- Table 4:

- Title:

- Source/permission status/credit line:

- Table 5:

- Title:

- Source/permission status/credit line:

- Table n, etc. $2^{2}$

\section{Figures}

\section{- Figure 1:}

${ }^{2}$ Add or delete items as necessary. Include a permission-status statement describing whether copyright permission is needed and, if so, whether it has been obtained. Include a credit line for each table that is reproduced from another source, even from your own previously published work. 
- Label:

- Filename:

- Source/permission status/credit line:

- Figure 2:

- Label:

- Filename:

- Source/permission status/credit line:

- Figure 3:

- Label:

- Filename:

- Source/permission status/credit line:

- Figure 4:

- Label:

- Filename:

- Source/permission status/credit line:

- Figure 5:

- Label:

- Filename:

- Source/permission status/credit line:

- Figure n, etc. $3^{3}$

\footnotetext{
${ }^{3}$ Add or delete items as necessary. Include a permission-status statement describing whether copyright permission is needed and, if so, whether it has been obtained. Include a credit line for each figure that is reproduced from another source, even from your own previously published work. When submitting your final files for publication, please provide source image files (in .eps or .ps format) for all figures at a minimum resolution of $300 \mathrm{dpi}$.
} 